\title{
MANUFACTURING COST OPTIMIZATION OF PHOTOVOLTAIC ENTERPRISES BASED ON NEURAL NETWORK
}

\author{
Weiping $\mathrm{ZHANG}{ }^{1,2}$, Shuming $\mathrm{LI}^{2}$, Junfeng $\mathrm{YU}^{2}$, and Yihua MAO ${ }^{3 *}$ \\ ${ }^{1}$ Department of Electronic Information Engineering, Nanchang University, China \\ ${ }^{2}$ Binhai Industrial Technology Research Institute of Zhejiang University, Tianjin, China; \\ ${ }^{3}$ Zhejiang University College of Civil Engineering and Architecture, Hangzhou, China; \\ *E-mail:kxtbi1337@163.com
}

\begin{abstract}
How to reduce the cost of photovoltaic power generation is the core issue of the survival and development of photovoltaic enterprises. Based on this, the manufacturing cost optimization of photovoltaic enterprises is studied based on neural network. Through the design of cost accounting control of photovoltaic enterprises, a genetic algorithm is proposed to optimize the manufacturing cost of photovoltaic enterprises, which is predicted at the maximum power point of the same photovoltaic power generation system. The results show that the RBF neural network optimized by genetic algorithm not only improves the prediction speed, but also improves the prediction accuracy. Thus, the maximum power point tracking control of photovoltaic power generation can be achieved better, and the manufacturing cost of photovoltaic enterprises can be optimized.
\end{abstract}

Keywords: RBF neural network (Radial basis function network), EPR system database, analytic network process (ANP), photovoltaic enterprises, optimization of manufacturing cost

\section{INTRODUCTION}

The development of photovoltaic industry is in its infancy. The idea of reducing the cost of products is mainly to reduce product costs through the revolution of new technologies and the development of new materials, which reduces the overall cost from the previous 10 yuan/ $\mathrm{W}$ to the current 4 yuan/ $\mathrm{W}$, and realizes the unit cost of electricity generation 0.8 yuan per $\mathrm{kWh}$ contribution [1]. This change plays a vital role in promoting the rapid development of the photovoltaic industry, and thus bringing about the new energy revolution and solving the environmental pollution problems of the traditional energy [2]. However, in the next few years, the development speed of new technology and new materials will slow down. In order to support the continued decline in PV generation costs needs to open up new space for reduction. The study found that through the refinement of the cost management and control of photovoltaic companies, relying on the internal management of enterprises to promote cost reduction and efficiency increase, is an important support point for the reduction of photovoltaic costs in the future. The most basic work of cost control is scientific and effective cost accounting analysis. Through accounting and analysis, there is a waste in the company's operations, which in turn drives cost improvement [3]. Therefore, the following research questions are put forward and solved through the cost accounting operation in photovoltaic enterprises. Reducing manufacturing costs is a management act. Only by identifying and improving the project on the basis of cost accounting and analysis can the cost be optimized and controlled. The main research area is the cost accounting application of photovoltaic enterprises. Through the combination of ERP system and the application of two cost accounting methods (standard cost method and activity-based costing), 
the cost data can be exported timely and effectively, and the waste in the cost is analyzed [4].

\section{STATE OF THE ART}

After systematically studying the relevant literature, it is found that the study of standard cost method and activity-based costing by domestic and foreign scholars focuses on the analysis of the method [5]. Standard cost method is widely used in enterprises, and most enterprises use this method in accounting costs. However, the shortcomings of the standard cost method are very prominent, which is not conducive to the company's comparison of manufacturing links and products. Moreover, the cost accounting cycle is long, and enterprises respond slowly to cost improvement. The operating flow of the activity-based costing method is complex, and the process involved is many and complex. It is difficult for general enterprises to carry out this work in operation [6]. ERP system in the current environment, the application of enterprises is relatively popular [7]. However, most companies mainly use the software in simple invoicing management, and simple standard costing has been used. Most studies only analyze problems in a single field, ignoring the integration of cost management. Empirical research has great limitations, poor replicable ability, inability to intuitively guide companies to solve practical problems, and lacks a comprehensive conceptual framework that integrates existing theoretical research and empirical research [8]. There are few cases of integration and integration of several cost management theories, and lack of analysis and use of information means, especially advanced ERP systems, to optimize the depth of cost management. For how to effectively combine the advantages of the standard cost method and the activity-based costing method under the ERP system, it is relatively less to develop a simple method of cost accounting and cost analysis. In particular, there is little research on the cost management of photovoltaic products and process characteristics.

\section{METHODOLOGY}

\subsection{PV Enterprise Manufacturing Cost Accounting Control Design and its Optimization Algorithm}

The algorithm of direct material cost in the standard cost method is: For example, if A product is
10 pieces and the direct material cost is 500 yuan, then the direct material cost per unit A product is $500 / 10=50$ yuan. The disadvantages of this algorithm are: first, the business unit only knows the cost of the unit material 50 yuan / blocks, it is difficult to know which material is abnormal. The second is the long accounting cycle, which requires financial data to be available after the financial closing at the end of the month. Thirdly, there are many influencing factors of direct material cost, which are caused by fluctuations in raw material prices and the actual consumption of materials and the change of standard dosage. The combination of factors in direct material costs makes it difficult to find improvements in the above financial data. Unit direct material cost $=$ art material unit price * (unit material theory consumption + excess loss dosage). Manufacturing costs consist of three parts: direct materials, direct labour and manufacturing costs. When calculating the manufacturing cost, the standard cost method takes the product as the accounting object, and obtains the unit manufacturing cost through the total manufacturing cost divided by the output quantity. The activity-based costing method is based on the standard costing method to increase the calculation of the process costs. In designing the manufacturing cost accounting of A PV enterprises, the following requirements are realized:

- First, the manufacturing cost data must be reported every day;

- Second, it is necessary to account for the manufacturing costs of different products, different times, different production workshops, and different sales orders.

To achieve the first requirement, it is necessary to report the data from time to time, and to respond to every ERP data in a timely manner through the use of the ERP system.

The implementation of the second requirement requires the addition of cost accounting objects and the introduction of the concept of work order here. The work order is the production instruction issued by the enterprise to the production department according to the sales order, which includes production workshop, production time, and material list and so on. At the same time, it is also used as a carrier for ERP system to issue materials and warehouse [9]. Therefore, according to the design of manufacturing cost accounting of A PV enterprises, the manufacturing cost accounting of unique work orders is constructed through the ad- 
dition of processing orders as accounting objects [10]. In addition, the traditional cost accounting is relatively simple for the apportionment of the amortization cost, such as the direct labour and the manufacturing cost, and is generally distributed directly according to the quantity of the products. Due to the different labour costs and processes of different products, the simple distribution according to the amount directly leads to the unreality of cost accounting. For example, the direct labour cost, the traditional algorithm is that the total labour cost for the month is 10,000 yuan, the monthly output A product is $4000 \mathrm{~W}$, the $\mathrm{B}$ product is $1000 \mathrm{~W}$, and the unit cost per $\mathrm{W}=10000$ / $(4000+1000)=2$ yuan/W. The drawback of this accounting method is that the default products $\mathrm{A}$ and $\mathrm{B}$ and the cost per unit of labour per unit is the same. In fact, it is possible that the employment cost of the product $\mathrm{A}$ is 1.5 times that of the product $\mathrm{B}$. According to the above algorithm, the unit cost calculated is the cost underestimation of product $\mathrm{A}$, and the cost of product $\mathrm{B}$ is overestimated. By analogy, the above problems also exist in the allocation of manufacturing costs. When designing the manufacturing cost of a work order, the following principles should be followed. First, in the work order cost report, the sales order corresponds to the corresponding production work order, which truly reflects the material picking cost of each production work order. Second, it is necessary to standardize the ERP operation of the enterprise and to enter the system data in real time. Third, according to the product's different processes and differences, design and share standards, truly reflect the characteristics of the product. After determining the weights of various processing parameters, the optimal processing cost can be determined by the following function:

$$
F(x)=\min \sum_{j=1, \ldots, n}^{i=1, \ldots, m}\left(P c_{I J} X_{I J} C_{I J} / \sum P c_{I J}\right),
$$

where $m$ is the total number of processing processes, that is, the total number of characteristics of substitute processing, and $n$ is the number of feasible processing methods corresponding to each process. $P C_{I J}$ is the weight of the processing parameter to the total cost calculated by ANP, and $C_{I J}$ is the corresponding cost of the processing technology. $X_{I J}$ is a decision variable for processing methods:

$$
X_{I J}=\left\{\begin{array}{l}
1, \text { Perform this processing } \\
0, \text { Do not perform this processing }
\end{array} .\right.
$$

And the processing of a feature must meet the requirement of machining accuracy.

$$
\sum_{j=1, \ldots, n} X_{I J} P q_{I J} \geq \eta P q_{I}
$$

Among them, $P q_{I J}$ is the accuracy coefficient corresponding to each processing process, and $P q_{I}$ is the characteristic $i$, the minimum precision coefficient required to achieve the processing, $\eta$ is the weight of machining accuracy corresponding to different reliability requirements. This is a combinatorial optimization problem. This type of problem has a very precise mathematical description, high computational complexity, and is arsenic. The conventional methods for solving combinatorial optimization problems include problem transformation, branch and bound method, hill climbing method and so on. When the problem size is small, applying these methods generally can get better feasible solution. When the size of the problem increases, the number of feasible solutions increases exponentially, leading to the combined explosion of search space. In actual production, the processing parameters that affect the total cost are often complicated, and the solution by traditional methods is no longer effective. Here a genetic algorithm is chosen to solve this problem. To determine the Group Scale (integer) and the algebra (integer) of the genetic operation, the initial algebra $\mathrm{k}=1$, using the random method or other methods to produce the $n$ possible solutions $X(1 \leq i \leq n, k=1)$ to form the initial solution group.

For each individual $X_{t}(k)$ in the group, the fitness $f\left(X_{t}(k)\right)$ is calculated. For each individual $X_{t}(k)$ in a group, the probability of survival is $P_{t}(k)$. According to the survival profile $P_{t}(k)$, the parent is selected to perform genetic operations in the group, including replication, crossover, and variation to obtain a new solution group. At this point $k=k+1$, the condition is satisfied and the operation ends. Compared with traditional optimization methods, genetic algorithm has the following characteristics:

- Genetic algorithm does not directly deal with the design variables of the problem itself, but the 


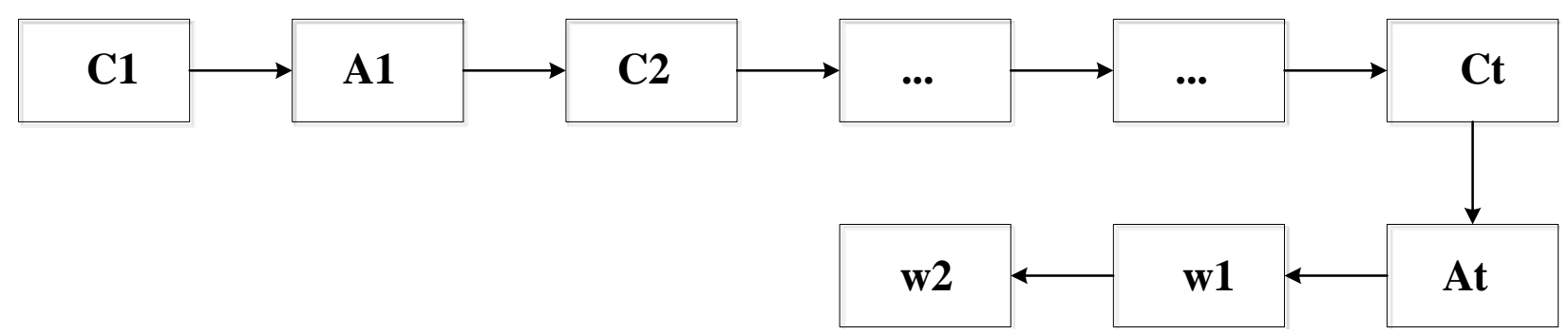

Fig.1. Genetic algorithm chromosome structure

coding of the design variables, which improves the universality of the algorithm;

- The search process of genetic algorithms begins with an initial group, rather than from a single individual, and has a parallel search feature, which greatly reduces the possibility of falling into the local optimal;

- Genetic operations used by genetic algorithms are all random operations. At the same time, it searches according to individual adaptation information and does not need other derivative information, so it has wide adaptability;

- Genetic algorithm has global search ability, has a strong and high computational efficiency, and is good at solving complex problems such as combination optimization;

- The genetic algorithm interacts with the same generation or the front and back groups through proper genetic manipulation to maintain the information that has been searched, which is incomparable to the optimization method based on the single search process.

\subsection{Genetic Algorithm for Optimizing RBF Neural Network}

The output layer, hidden layer, and RBF neural network output layer have different functions. When designing the network, the most critical step is the hidden layer data centre $C_{i}$ expansion constant $\delta_{i}$ and the choice of $w$ between the hidden layer and the output layer. Only by selecting the most accurate parameter values the RBF neural network can get the best approximation effect. Therefore, the genetic algorithm is used to optimize the network, that is the best choice of the three parameters. The specific optimization steps are as follows: select chromosomes and encode them. RBF network design is generally divided into two independent steps. First, the data centre ink stone and the expansion constant of the network are selected through the $k$-means clustering method, and then the weight $w$ is solved through the least square method. In this way, the required solution is computed, which is not conducive to the unity of the whole algorithm. The genetic algorithm is encoded by the three parameters, such as $C_{i}, \delta_{i}$, and $w_{i}$; and the chromosome string is formed. The data centre of the hidden layer plays an important role in the input and output layer in the radial basis function. Because the base function of RBF neural network is Gauss's function, the changes of two parameters of data centre $C_{i}$ and extension constant $\delta_{i}$ Bare closely related, and the change of data centre will affect the extension constant change. Therefore, the genetic algorithm will intersect two parameters of $C_{i}$ and $\delta_{i}$, that is, a data centre is composed of a set of expansion constants. Then put the weights $\mathrm{w}$ in the last order, which can increase the probability that data centres and extension constants will change simultaneously in the genetic process. The chromosome structure of the genetic algorithm is shown in Fig. 1.

If the number of hidden layer neurons in the RBF neural network is $P$, the number of input layer neurons is $M$, and the number of output layer neurons is $N$, the number of weights is $M N$. The data centre and width are $P N+P$, and the total length of chromosomes is $M N+P N+P$. In addition, the number of neurons in the hidden layer will vary

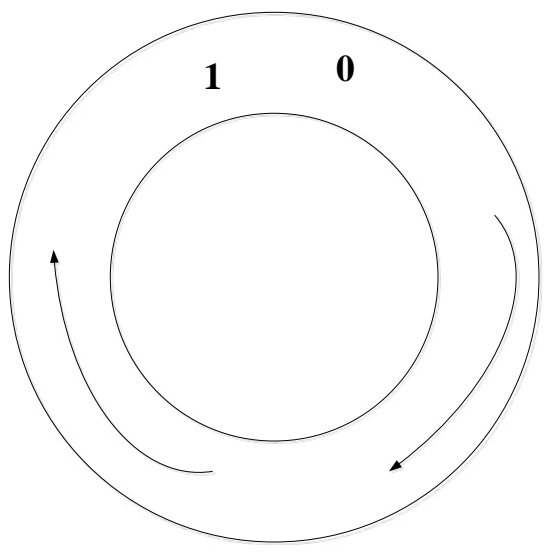

Fig.2. Intersection and selection diagram in genetic algorithm 
Table 1. Before and After Improvement

\begin{tabular}{|c|c|c|c|}
\hline & Manufacturing cost, yuan/W & Overall cost, yuan/W & Profit \\
\hline 2012 & 3.8 & 45000 & $12 \%$ \\
\hline 2013 & 3.6 & 42000 & $14 \%$ \\
\hline Ring than & $5 \%$ & $7 \%$ & $17 \%$ \\
\hline
\end{tabular}

with the approximation ability of the RBF neural network in the course of heredity. The ratio of fitness calculated to each individual corresponds to each other. During the random number is generated in the range of $[0,1]$, the number that the pointer points will be selected. The schematic diagram of the selection operation is shown in Fig.2. In this operation, the higher fitness of individuals is higher probability of pointer selection, which indicates that the three parameters corresponding to $C_{i}, \delta_{i}$ and $w_{i}$ are better.

\section{RESULT ANALYSIS AND DISCUSSION}

\subsection{Data acquisition and preprocessing}

In this part, we use the algorithm and model to calculate. The specific idea is: firstly, we get the data from photovoltaic enterprises, and summarize the data, then compare the two numerical prediction methods, get the best method, and finally carry out numerical prediction.After introducing new cost accounting schemes, PV enterprises get good profits in the actual business operation. XX enterprises formally introduces the cost management plan in 2013, and achieves better returns from the completion of various economic indicators, as shown in Table 1.

As shown in Table 1, the cost of manufacturing is down by $5 \%$. In 2012, the manufacturing cost was 3.8 yuan per watt. In 2013, the manufacturing cost was 3.6 yuan per watt, which was $5 \%$ lower than the previous quarter. In order to make the data more comparative, the same material prices as in 2012 were used to account for 2013 manufacturing costs for the study). Four major costs (financial costs, administrative costs, $R \& D$ expenses, sales costs). The ring fell $7 \%$, and the gross profit increased by $17 \%$. From the above economic data is not difficult to obtain, there is a significant improvement before and after the import. A real and effective accounting method brings enterprise management with the exception of quantitative data. More derivative benefits can be summed up as four points: cost accounting

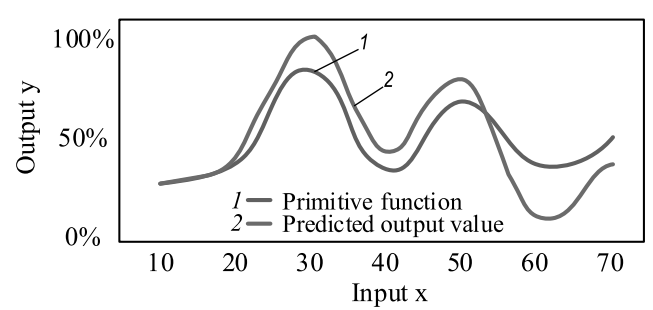

Fig.3. RBF neural network output graph based on randomly selected centre method

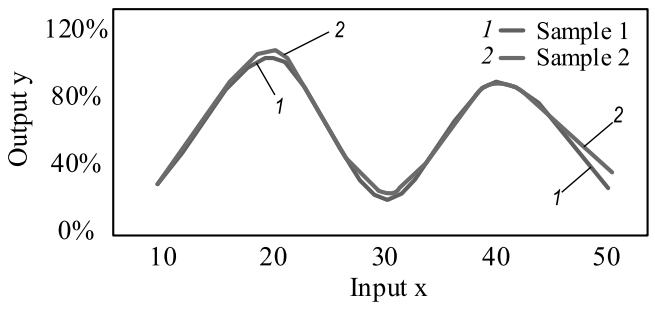

Fig.4. RBF neural network output graph based on k-means clustering

and business operation. Costing is based on a principle of consistency with business processes. According to the different products, the real allocation coefficient is used to ensure that the accounting data are not distorted. In this way, the acceptance of business units is relatively high.

\subsection{Numerical prediction}

Then we choose K-means clustering method and random center selection method to simulate the function, and compare the results to confirm the most suitable function simulation method.

The random selection centre method can randomly select data as data centre through random number, then the data centre is fixed and its corresponding extension constant is no longer changed. Then the weights are determined by the least square method. Fig. 3 shows the RBF neural network's ability to approximate the function after training based on the randomly selected centre method. The dotted curve is the original function, and the solid curve is the predicted output value of the network. 
Table 2. Partial RBF Neural Network Training Data

\begin{tabular}{|c|c|c|c|c|}
\hline Time & $\mathbf{W} / \mathbf{m}^{\mathbf{2}}$ & ${ }^{\mathbf{0}} \mathbf{C}$ & $\mathbf{V}$ & $\mathbf{W}$ \\
\hline 6.083 & 63.4720 & 25.2756 & 28.9800 & 9.9357 \\
\hline 6.167 & 103.066 & 25.3657 & 29.2500 & 19.0074 \\
\hline 6.250 & 87.6348 & 25.4578 & 29.5600 & 15.2387 \\
\hline 6.330 & 127.8117 & 25.4135 & 29.9400 & 23.4470 \\
\hline
\end{tabular}

After initializing the network, the samples are categorized into different categories in order of distance from near to far. Save old cluster centres and generate new cluster centres. The data centre is determined by comparing whether the two central locations coincide and the weight is determined by least square method. The predicted output is shown in the curve in Fig. 4.

From the comparative analysis of Figs 3 and 4, it is shown that the $k$-means clustering method can simulate the original function more accurately than the random selection centre method. There is a close relationship between the prediction degree of RBF neural network and the number and location of the data centre, and the random selection centre method is more random to the data centre. Therefore, $k$-means clustering and least square method are used to determine the data centre and weights of RBF neural network. After choosing the appropriate function simulation method, this paper uses the random center selection method to carry out function simulation and numerical prediction. In the numerical prediction, we first normalize the target vector, and then predict.

The selected target vector is $\mathrm{X}=[($ time $(\mathrm{Ct})$, light intensity (Sun), temperature $\left.\left(\mathrm{T}_{\mathrm{ac}}\right)\right]$, while the counter normalized output vector is required to be the corresponding voltage of the maximum power point. Part of the training data and prediction data of the network are shown in Table 2.

A total of 145 sets of data for forecasting PV MPPT were collected from 6:00 a.m. to 18:00 p.m. on May 2, 2013. The average is divided into one group every 5 minutes. The first 100 groups are the training data of the RBF neural network, and the other 45 groups are the prediction data of the network. After the input data is determined, the centre and weights of the RBF neural network are determined by the $k$-means clustering method and the least square method. Select the first 100 sets of data as samples to input data for training, and then use the 45 sets of data to predict, in order to test the accuracy and practicability of the network. The result of network prediction is the voltage corresponding to the maximum power point in photovoltaic generation when the time, intensity and temperature are changing.

\section{CONCLUSION}

The development of photovoltaic industry is in its infancy. On the way of lowering the cost of products, it is mainly through the revolution of new technology and the development of new materials to reduce the cost of products and reduce the overall cost. This change plays a vital role in solving the problem of environmental pollution of traditional energy sources. Based on this, the manufacturing cost optimization of photovoltaic enterprises is studied based on neural network. First of all, the status and problems of the research are expounded. Secondly, based on neural network algorithm, this paper puts forward the design and optimization algorithm of manufacturing cost accounting control of photovoltaic enterprises, and then takes the A enterprise as an example to test it. The models and algorithms can be effectively applied in enterprises. Only by identifying and improving the project on the basis of cost accounting and analysis can the cost be optimized and controlled. Through the combination of ERP system and the application of two cost accounting methods (standard cost method and activity-based costing), the cost data can be output in time and effectively, and the waste in the cost is analyzed.

\section{REFERENCES:}

1. Baptista D, Abreu S, Travieso-González C, et al. Hardware implementation of an artificial neural network model to predict the energy production of a photovoltaic system. Microprocessors and Microsystems, 2017. V49, pp.77-86.

2. Dumitru C D, Gligor A, Enachescu C. Solar photovoltaic energy production forecast using neural networks. Procedia Technology, 2016. V22, pp.808-815.

3. Kayri I, Gencoglu M T. Predicting power production from a photovoltaic panel through artificial neural networks using atmospheric indicators. Neural Computing \& Applications, 2017, pp.1-14.

4. Kane P V, Andhare A B. Application of psychoacoustics for gear fault diagnosis using artificial neural 
network. Journal of Low Frequency Noise, Vibration and Active Control, 2016. V35, \#3, pp.207-220.

5. Zheng M, Horowitz K, Woodhouse M, et al. IIIVs at scale: a PV manufacturing cost analysis of the thin film vapour-liquid-solid growth mode. Progress in Photovoltaic: Research and Applications, 2016. V24, \#6, pp.871-878.

6. Haiying Li, Xian Li, Xinyue Xu, Jun Liu, Bin Ran. Modelling departure time choice of metro passengers with a smart corrected mixed logit model - A case study in Beijing. Transport Policy. 2018, \#69, pp.106-121.

7. Moon S, Yoon S G, Park J H. A new low-cost centralized MPPT controller system for multiply distributed photovoltaic power conditioning modules. IEEE Transactions on Smart Grid, 2015. V6, \#6, pp.2649-2658.
8. Jain S, Agarwal V. A single-stage grid connected inverter topology for solar PV systems with maximum power point tracking. IEEE transactions on power electronics, 2007. V22, \#5, pp.1928-1940.

9. Goti-Elordi, Aitor; de-la-Calle-Vicente, Alberto; Gil-Larrea, Mara-Jose, et al. Application of a business intelligence tool within the context of big data in a food industry company. DYNA, 2018. V92, \#3, pp. 347-353.

10. Oman S, Leskovar R, Rosi B, et al. Integration of MES and ERP in supply chains: effect assessment in the case of the automotive industry. Tehnički vjesnik, 2017. V24, \#6, pp.1889-1896.

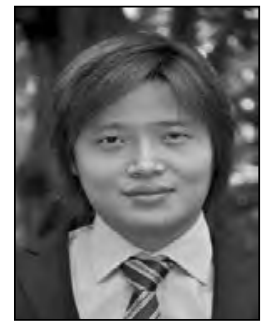

Weiping ZHANG

received the Ph.D. degree in computer science from Rostock University, Rostock, Germany. From 2014 to 2016, he was a Research Scientist with the Institute of BCRT in Berlin, Humboldt University, Berlin, Germany. He is currently a Uni-Professor with the School of Information Engineering, Nanchang University, Nanchang, China. His research interests include machine learning, process information management systems and real-time mobile measurements of physiological parameters

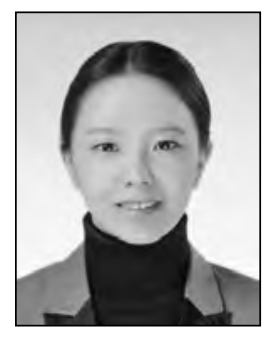

Shuming LI, Post Dr. She has received Ph.D. degree in Beijing Forestry University in 2013. From 2017 till now, she began her postdoctoral research at Binhai Industrial Technology Research Institute of Zhejiang University. Her research interests include high value utilization of biomass, technology innovation management and commercialization of research findings

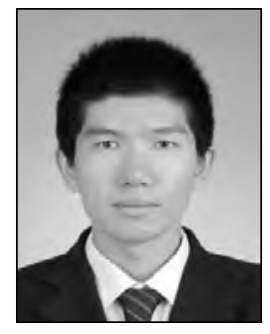

Junfeng $\mathbf{Y U}$ received the master's degree in communication and information system from Beijing Jiaotong University, Beijing, China. He has more than 5 years development and operation experience in the internet industry, particularly in domain name system and routing technology. He is currently an R\&D engineer in Binhai Industrial Technology Research Institute of Zhejiang University, Tianjin, China. His research interests include machine learning and data analysis

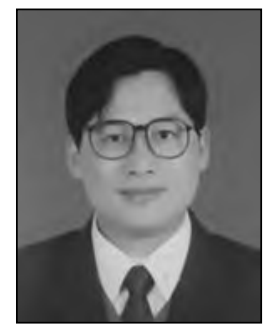

Yihua MAO, Ph.D, Professor. He has got Ph.D. degree from Zhejiang University. Now he serves in College of Civil Engineering and Architectural of Zhejiang University. He also serves as the deputy dean of Industrial Technology Research Institute of Zhejiang University and the dean of Binhai Industrial Technology Research Institute of Zhejiang University. His main research fields: the engineering economy and project cost, construction project management, system design and large data analysis technology; enterprise strategy and technology innovation management 